\title{
Grain size effect on transferability in micro-coining process assisted by ultrasonic vibration
}

\author{
Tetsuhide Shimizu ${ }^{1, *}$, Shingo Kosuge ${ }^{1}$, and Ming Yang $^{1}$ \\ 1 Division of Human Mechatronics Systems, Graduate School of System Design, Tokyo Metropolitan University, Japan 6-6, \\ Asahigaoka, Hino-shi, 191-0065 Tokyo, Japan
}

Received 7 February 2015 / Accepted 24 February 2015

\begin{abstract}
The present study reports the assisting effect by the ultrasonic vibration in a micro-coining process. Particularly, we were focused on the grain size of the work material to study the scale dependency of transferability under the assistance of ultrasonic vibration. The pure copper plate of $0.3 \mathrm{~mm}$ in thickness was used as the working material. The micro-coining process was performed on the specimens with three different grain sizes which were obtained through an annealing process. The result showed that the vibration assisted effect on transferability becomes more significant for the material with larger grain sizes. Assisting effect of ultrasonic vibration for the coarse grained materials is also demonstrated.
\end{abstract}

Key words: Microforming, Ultrasonic vibration, Grain size effect, Micro-coining, Transferability

\section{Introduction}

With increasing demand for the miniaturization and higher-functionalization of micro-devices, the high efficient with high accurate manufacturing method is strongly required from the industry [1]. Microforming of metallic materials is one of the solutions to satisfy these demands. In construction of the process design of microforming, which produces the microparts with a few-tens of $\mu \mathrm{m}$ to sub-mm scale dimensions, an industrial challenge confronted with the technical problems due to the effect of the miniaturization of conventional metal forming, which is called size effect [2].

In particular, since the polycrystalline metallic alloys are largely used as working materials, the effect of grain size is an important factor, due to that the relative volume of each grain becomes relatively larger with down scaling. Due to the inhomogeneous material flow of the individual grains, the controllability of material deformation becomes more difficult with a miniaturization of the process dimensions [3]. Therefore, the dimensional accuracy becomes worse due to this inhomogeneous material flow during the process. Additionally, in micro-forging and -coining process, since the enormous forming load is required to improve this inhomogeneous material flow, the overloading to the forming die and tools may shorten its tool life [4].

In order to improve this inhomogeneous material flow and to reduce the overload on the forming tools, a number of

*e-mail: simizu-tetuhide@tmu.ac.jp studies from the aspect of material developments have been performed, e.g. application of superplastic materials [5] and bulk metallic glasses [6] etc. While, from the view point of process developments, authors have been applied the additional high-dense energy during the process, such as vibration impact or the material heating [7-11]. In the previous report, the effect of ultrasonic vibration on surface smoothening [7-9] and its transferability of $\mu \mathrm{m}$-scale $\mathrm{V}$-shaped groove have been clarified [10].

For the further investigation on the transferability in microcoining process and to clarify the range of application for the assisting effect of ultrasonic vibrations, the present study reports the applicability to the coarse grained materials, which has not been successfully transferred, due to its dominant material flows of individual grains. To discuss its grain size effect on micro V-groove transferability in material flows under ultrasonic vibration, the micro-coining process is performed to the copper plate with three different grain sizes and its transfer height, coining force, and surface roughness are evaluated in detail.

\section{Experimental}

\subsection{Materials}

The materials used are pure copper plate with a thickness of $0.3 \mathrm{~mm}$. To obtain the specimens with different grain size, the as-received material are annealed with temperature of 300 


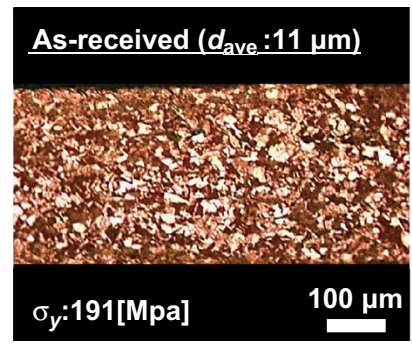

(a)

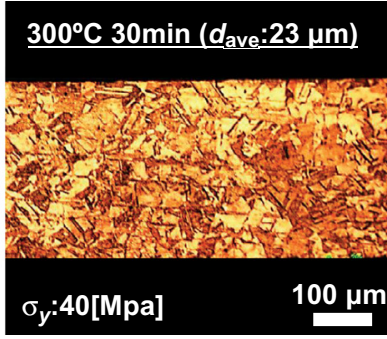

(b)

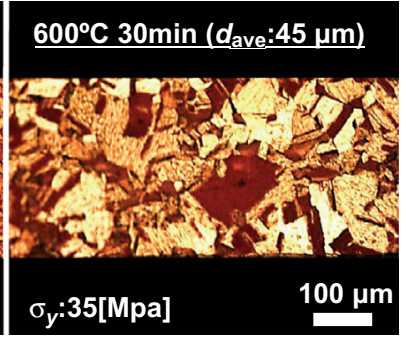

(c)

Figure 1. Optical microscope images of microstructure of pure-copper plate with different annealing conditions (a) as-received, (b) $300{ }^{\circ} \mathrm{C}$ 30 min annealed, (c) $600{ }^{\circ} \mathrm{C} 30$ min annealed.

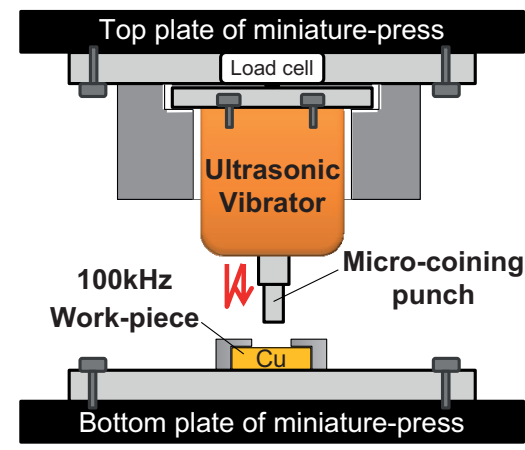

(a)

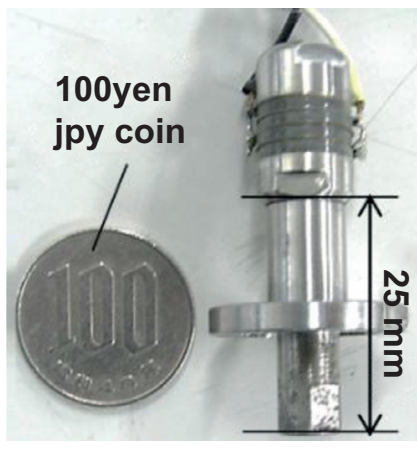

(b)

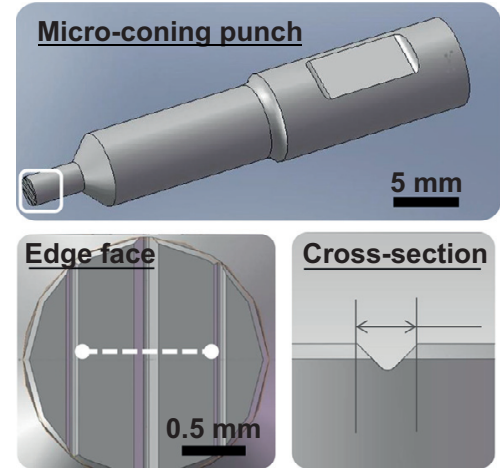

(c)

Figure 2. Schematic illustration of ultrasonic vibration assisted micro-coining system (a) construction of ultrasonic vibration microforming system, (b) appearance of ultrasonic vibrator, (c) schematic of micro-coining punch.

and $600{ }^{\circ} \mathrm{C}$, which is the higher temperature than the recrystallization temperature of $200{ }^{\circ} \mathrm{C}$ for pure copper, for $0.5 \mathrm{~h}$ at the nitrogen atmosphere. Figure 1 shows the microstructure images of as-received, 300 and $600{ }^{\circ} \mathrm{C}$ annealed pure copper specimens, obtained by the optical microscopy. The average grain size for each samples are 11,23 , and $45 \mu \mathrm{m}$ for as-received, 300 and $600{ }^{\circ} \mathrm{C}$ annealed specimens, respectively. In particular, the coarse grained structure with approximately 4-10 grains through the thickness was successfully obtained by the $600{ }^{\circ} \mathrm{C}$ annealing process. Additionally, the Vickers hardness measurements were performed to calculate the yield stress, $\sigma_{\mathrm{y}}$, for each sample after the heat treatments. The $\sigma_{\mathrm{y}}$ values calculated for each sample are indicated also in Figure 1.

\subsection{Experimental setup}

Ultrasonic vibration assisted micro-coining experiments were performed with the experimental systems developed by authors in the previous report [10]. This system can be installed inside the compact space of desktop size precision press machine developed by the authors [12]. To apply the highly-dense energetic vibration during the forming process, the compact ultrasonic vibrator with the frequency of $100 \mathrm{kHz}$ was used instead of the $20 \mathrm{kHz}$ vibrator for the conventional ultrasonic vibration assisted metal forming. This vibrator can realize the maximum vibration amplitude of $2 \mu \mathrm{m}$ even with the compact dimensions of $25 \mathrm{~mm}$ in length. Figure 2a illustrates the ultrasonic vibration assisting system as mentioned. At edge of the ultrasonic vibrator, micro-coining punch with a diameter of $2 \mathrm{~mm}$ was attached as shown in Figure 2c. The dimensions of the micro coining punch were specially designed to transfer the maximum amplitude from the vibrator to the edge of the micro-coining punch. At the edge-face of the coning punch, the V-shaped groove with a width of $200 \mu \mathrm{m}$, a depth with $90 \mu \mathrm{m}$, edge angle with $90^{\circ}$ and with the corner radius of $30 \mu \mathrm{m}$ was prepared by precision machining process. Micro-coining punch was made of sintered WC-Co hard alloy (JIS: V20 tungstencarbide-cobalt alloy). The obtained average surface roughness at the V-grooved surface was $0.03 \mu \mathrm{mSa}$.

In the micro-coining experiments, the ultrasonic vibrations were applied after the punch load approached the $50 \mathrm{~N}$ and continued until the certain displacement of the conditions. The coining speed was set to $0.01 \mathrm{~mm} / \mathrm{s}$ and the forming load on the punch were measured by load-cell attached above the coning punch. To investigate the difference in the transferability depending on the material grain size, the maximum transfer height, coining force, and the surface roughness at the slope of the transferred surface are evaluated in detail. For the transfer height measurements, a non-contact three dimensional profiler (Talysurf, CCI, Taylor Hobson, UK) was used. In addition, the surface roughness was measured by using confocal laser scanning microscopy (OLS300, Olympus, Japan) was used. Moreover, the scanning electron microscopy (SEM, VE8800, 


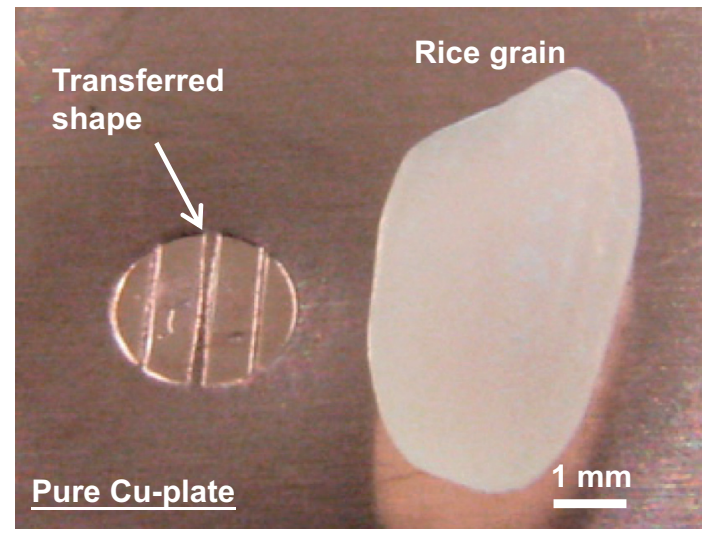

Figure 3. Appearance of transferred shape after micro-coining.

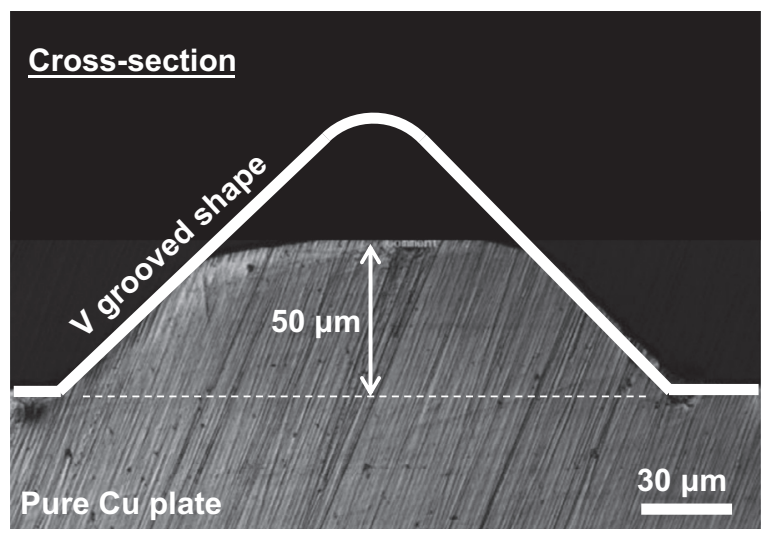

Figure 4. Optical micrographs of cross-section of transferred height.

Keyence, Japan) was used for the observation of the surface morphology at the sloped surface.

\section{Results and discussion}

\subsection{Maximum transfer height}

Figure 3 shows the appearance of the pure copper sample after the micro-coining process. In comparison with the scale of the rice grain, it can be confirmed that the tiny round shape of the coning punch and V-groove are successfully transferred to the pure copper surface. In order to compare the maximum transfer height between the different grain size materials under the same coining load, which can obtain the transfer height with $50 \mu \mathrm{m}$ without ultrasonic vibration (see Figure 4) was applied for all specimens. Figure 5 compares the increasing ratio of transfer height to the maximum transfer height without ultrasonic vibration in different grain size conditions. The increasing ratio of transfer height, $H_{\text {tr }}$ was defined by a following equation (1):

$$
H_{\mathrm{tr}}=\frac{h_{\mathrm{uv}}-h_{\mathrm{nv}}}{h_{\mathrm{nv}}},
$$

where $h_{\mathrm{uv}}$ is the transfer height under the process with ultrasonic vibration and $h_{\mathrm{nv}}$ is that without ultrasonic vibration for

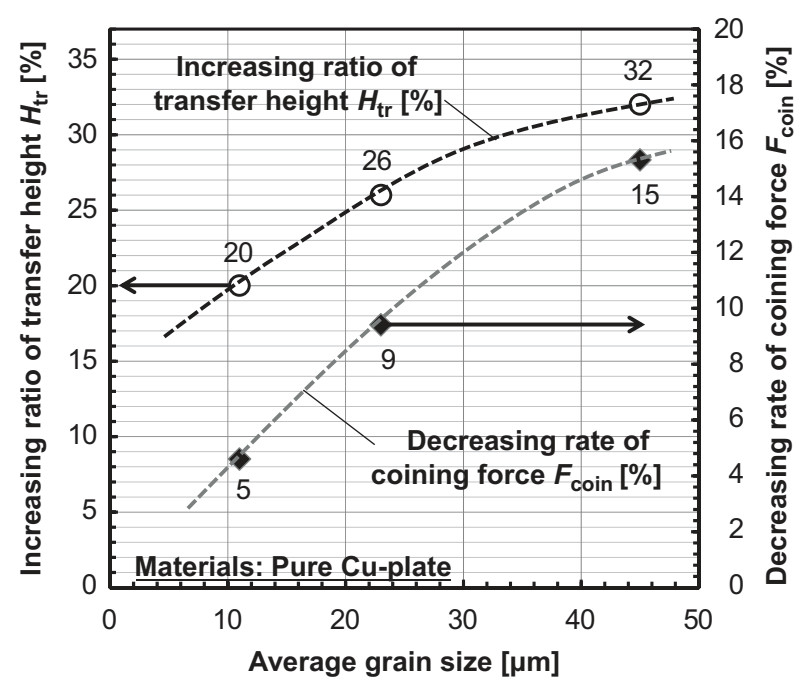

Figure 5. Comparison data of increasing ratio of transfer height and decreasing rate of coning force by applying ultrasonic vibration as a function of the average grain size of the pure copper samples.

each grain size condition. By dividing with the transfer height without ultrasonic vibration at corresponding grain size condition, the difference in yield stress between the different grain sizes can be eliminated.

As shown in Figure 5, with increasing the grain size, the increasing ratio becomes higher. Particularly, the coarse grained sample with $45 \mu \mathrm{m}$ indicates the highest increasing ratio of $32 \%$. This, in turn, suggests that the larger effect of ultrasonic vibration was obtained for coarse grained material. This may be attributed to the larger contact area of surface grains for coarse grained material, such as $45 \mu \mathrm{m}$ in grain size in the present study. Since the ultrasonic vibration is well known to activate the dislocation movement in the material, the release of the dislocation at the surface grain is further promoted with the assistance of ultrasonic vibration. Therefore, with increasing the grain size, this promoted area might become larger, resulting in the lower flow stress in microcoining process.

\subsection{Reduction of coining load}

As similar with the evaluation of maximum transfer height above, the coining loads, which can obtain the transfer height with $50 \mu \mathrm{m}$ with the ultrasonic vibration, were evaluated. In order to describe the effect of ultrasonic vibration efficiently, decreasing rate of coining force to the force without ultrasonic vibration was introduced. The decreasing rate of coining force, $H_{\text {tr }}$ was defined by a following equation (2):

$$
F_{\text {coin }}=\frac{f_{\mathrm{uv}}-f_{\mathrm{nv}}}{f_{\mathrm{nv}}}
$$

where $f_{\mathrm{uv}}$ is the transfer height under the process with ultrasonic vibration and $f_{\mathrm{nv}}$ is that without ultrasonic vibration for each grain size condition.

As shown in Figure 5, by applying the ultrasonic vibration during the forming process, the forming load decreased for all 

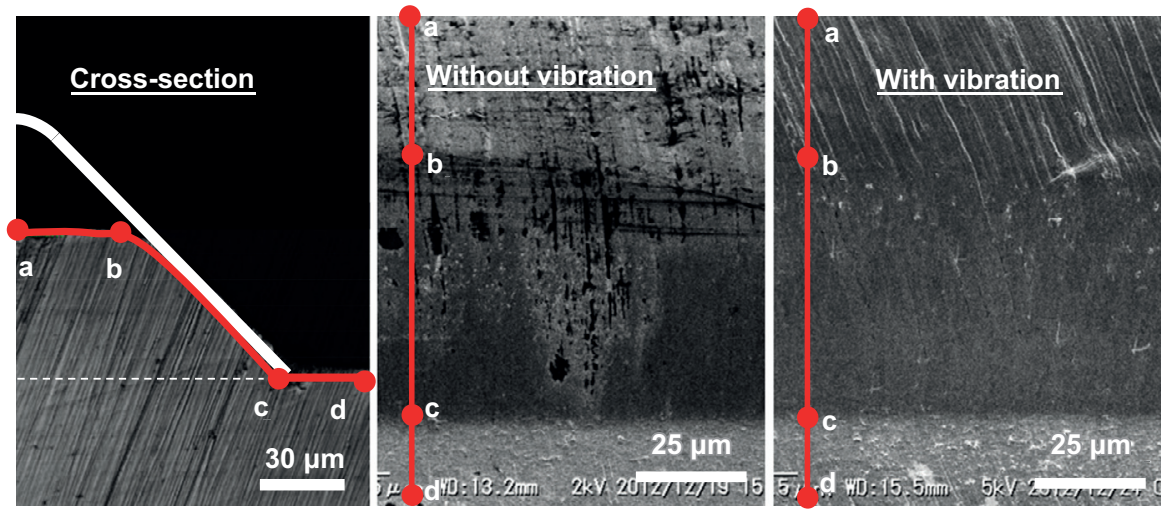

(a)

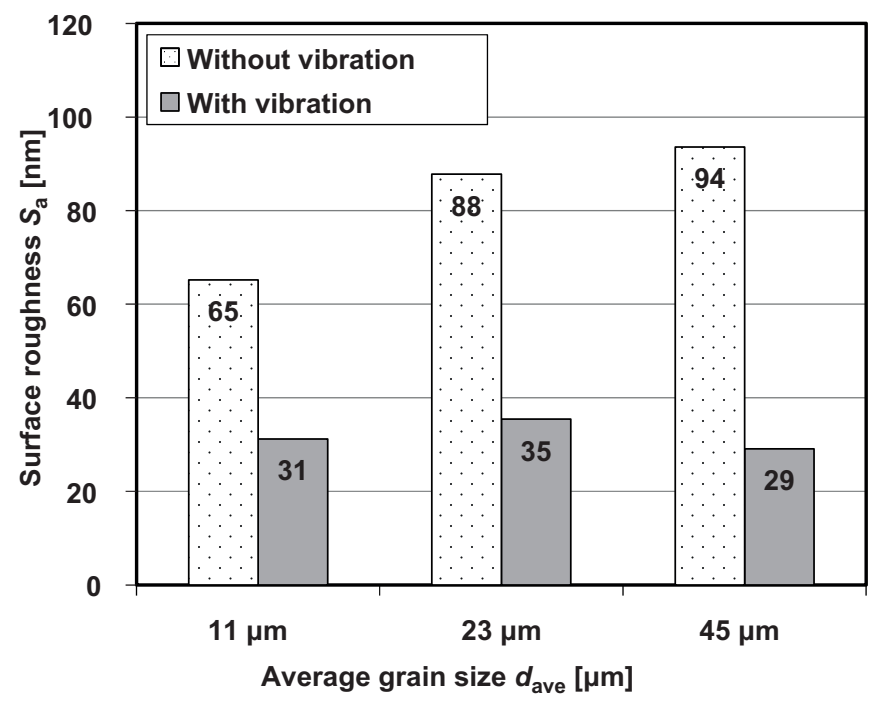

(b)

Figure 6. Effect of ultrasonic vibration on surface smoothening in micro-coining process (a) cross-section images and SEM surface images of sloped surface of $600{ }^{\circ} \mathrm{C}$ annealed sample transferred with and without ultrasonic vibration, (b) surface roughness after micro-coining with and without ultrasonic vibration for different samples with different average grain size of 11,23 , and $45 \mu \mathrm{m}$.

samples. Additionally, with increasing the grain size, the decreasing rate by the ultrasonic vibration becomes more significant. As is well corresponded to the tendencies with the results of maximum transfer height, the largest decreasing rate was obtained for the sample with the grain size of $45 \mu \mathrm{m}$. Consequently, it was demonstrated that the ultrasonic vibration assisting effect is rather significant for the coarse grained material. As mentioned above, the larger contact area of surface grains for coarse grained material might contribute to enlarge the promoting area of dislocation movement at the contact surface. The increase of this soften area might contribute to lower the forming load during the micro-coining process.

\subsection{Surface roughness}

Figure 6a shows the cross-section and SEM observation images of the sloped surface of transferred V-shaped structure for $600{ }^{\circ} \mathrm{C}$ annealed sample. As denoted in Figure 6a, crosssection of a-b-c-d line is corresponded to the slope surface as shown in the right hand side. In comparison with the sloped surface without vibration, the surface with vibration indicates the relatively smooth surface at the contact area with $\mathrm{V}$ groove, which is corresponded to the line of b-c area. This effect on the surface smoothing is well corresponded to the results obtained in the previous reports [7-9]. In particular, the mechanism of the surface deformation under vibration assistance was further discussed in the reference [8].

Additionally, the surface roughness values at the sloped surface with and without ultrasonic vibration for the samples with different grain size are summarized in Figure 6b. It is confirmed that, for the all samples, the surface roughness decreases more than $50 \%$ by applying ultrasonic vibration. Moreover, although the surface roughness increases with increasing grain size under conditions without the assistance of the ultrasonic vibration, when the ultrasonic vibration is applied, the surface roughness value keeps constant with $0.03 \mu \mathrm{mSa}$, irrespective of the grain size. In fact, this surface roughness values are in good agreement with the initial surface 
roughness of the coining tools. That is to say, the micro topography of the tools was successfully transferred to the material by applying the ultrasonic vibration. Consequently, by applying the ultrasonic vibration, the surface roughening due to the inhomogeneous material flows of the individual grains is suppressed and the uniform material flow can be obtained with a smooth surface roughness.

\section{Conclusions}

To investigate the grain size effects on the transferability of micro-coining process assisted by the ultrasonic vibration, and to clarify the applicability of the effects of ultrasonic vibration assistance to the coarse grained materials, the micro-coining tests were performed on the pure copper sheets with different, average grain size of 11,23 , and $45 \mu \mathrm{m}$ respectively, from which the following conclusions may be drawn:

1. In comparison of the maximum transfer height, larger increasing ratio of $32 \%$ in transfer height by applying the ultrasonic vibration was obtained for the material with $45 \mu \mathrm{m}$ gain size.

2. Corresponding to the tendency of maximum transfer height, decreasing rate of the coining load was obtained for the same transfer height, for the material with a large grain size.

3. Surface smoothing effect from ultrasonic vibration is rather higher for the coarse grained material. Irrespective of the grain size of the material to be deformed, a similar smoothness to the forming tool-surface was achieved through applying the ultrasonic vibration.

\section{Implications and influences}

The present study clarified the assisting effect on the inhomogeneous material-flows of the coarse grained material from the ultrasonic vibration in micro-coining process. This study suggests there is higher assisting effect from ultrasonic vibration on the forming process when the process takes place at a smaller length-scale. This may contribute to the improvement of the forming accuracy of microparts, which would further assist in realization of further miniaturization and higherfunctionalization of micro-devices.

Acknowledgements. The authors gratefully acknowledge the funding from the Japan Society for the Promotion of Science (JSPS), for a Grant-in-Aid for Young Scientists B (No. 26820327), and from the AMADA foundation for a Grant-in Aid for general research and development (AF-2013028).

\section{References}

1. M. Geiger, M. Kleiner, R. Eckstein, N. Tiesler, U. Engel, Microforming, CIRP Ann.-Manuf. Technol. 50-2 (2001) 445-462.

2. F. Vollertsen, D. Biermann, H.N. Hansen, I.S. Jawahir, K. Kuzman, Size effect in manufacturing of metallic components, CIRP Ann.-Manuf. Technol. 58 (2009) 566-587.

3. H. Justinger, G. Hirt, Estimation of grain size and grain orientation influence in microforming processes by Taylor factor considerations, J. Mater. Process. Technol. 209 (2009) 2111-2121.

4. Y. Saotome, T. Zhang, A. Inoue, Microforming of MEMS parts with amorphous alloys, Mater. Res. Soc. Symp. Proc. 554 (1999) 385-390.

5. W.J. Kim, S.J. Yoo, H.K. Kim, Superplastic microforming of $\mathrm{Mg}-9 \mathrm{Al}-1 \mathrm{Zn}$ alloy with ultrafine-grained microstructure, Scr. Mater. 59-6 (2008) 599-602.

6. Y. Saotome, K. Imai, S. Shioda, S. Shimizu, T. Zhang, A. Inoue, The micro-nanoformability of Pt-based metallic glass and the nanoforming of three-dimensional structures, Intermet. 10 (2002) 1241-1247.

7. M. Yang, H. Tanabe, T. Shimizu, High-density energy assisted micro forming for fabrication of metallic devices, Proc. 15th Int. Conf. Adv. Mater. Process. Technol. (2012) (AMPT2012) CD-ROM.

8. Y. Bai, M. Yang, Investigation on mechanism of metal foil surface finishing with vibration-assisted micro-forging, J. Mater. Process. Technol. 213-3 (2013) 330-336.

9. Y. Bai, M. Yang, Optimization of metal foils surface finishing using vibration-assisted micro-forging, J. Mater. Process. Technol. 214-1 (2014) 21-28.

10. Kosuge S., Yang M., Improvement in processing accuracy of micro parts by ultrasonic vibration assisted forging, Proc. 15th Int. Conf. Adv. Mater. Process. Technol. (2012) (AMPT2012) CD-ROM.

11. Q. Zheng, T. Shimizu, T. Shiratori, M. Yang, Tensile properties and constitutive model of ultrathin pure titanium foils at elevated temperatures in microforming assisted by resistance heating method, Mater. Des. 63 (2014) 389-397.

12. T. Shimizu, M. Ogawa, M. Yang, K. Manabe, Plastic anisotropy of ultra-thin rolled phosphor bronze foils and its thickness strain evolution in micro-deep drawing, Mater. Des. 56 (2014) 604-612.

Cite this article as: Shimizu T, Kosuge S \& Yang M: Grain size effect on transferability in micro-coining process assisted by ultrasonic vibration. Manufacturing Rev. 2015, 2, 5. 